Vietnam Journal of Mechanics, VAST, Vol.33, No. 3 (2011), pp. $148-161$

\title{
MATHEMATICAL MODELLING AND CONTROL OF THE EVOLUTION OF DYNAMIC SYSTEMS INTERACTING WITH MEDIUM
}

\author{
V.L. Zakovorotny ${ }^{1}$, Nguyen Dong Anh $^{2}$, Pham Dinh Tung ${ }^{1}$ \\ ${ }^{1}$ Don State Technical University, Rostov-Don, Russia \\ ${ }^{2}$ Institute of Mechanics, Hanoi, Vietnam
}

\begin{abstract}
The article proposes a new method of mathematical modeling evolutional systems interacting with medium. A cutting process or tribo-space formed in the contact area of two conjugate mechanical subsystems is considered as medium. The features of the medium depend not only on state coordinates of systems but also on trajectories. It is obtained that the parameters of the medium are performed as integral operators of equations like Volterra equations of $2^{\text {nd }}$ type. The problems of control of evolutional systems are being analyzed.
\end{abstract}

Keywords: Evolutional system, interact, medium.

\section{INTRODUCTION}

Studying evolution of technical systems is traditional and corresponds to the problem of ensuring reliability. In particular, studying evolutional transformations of processes on cutting machines affects the development of wearing of instruments changing cutting products quality indexes [see e.g. 1-5]. All those indexes characterize the external display of evolutional changes. The evolutional changes of dynamic characteristics of cutting process, which influences the parameters of geometrical quality of goods and the state of cutting process, perform a more important meaning. They affect the current characteristics of the irreversible transformations, which are characterized by trajectories of changing the power of irreversible transformations of the function of work being performed. In technical systems with the friction areas the evolutional changing of matrices of dynamic rigidity and dissipation of tribo-space, being formed in the areas of conjugation of the contacted elements, takes place. The article is devoted to studying evolutional changes of the dynamic environmental characteristics, which allow correcting the control. The important point is that while implementing synergetic control conception the determination of variety of trajectories in the space of state plays the major role. This variety should characterize natural conditions of system functioning, however, the management in this case needs only to tune trajectories to necessary ones for normal functioning [6]. The article proposes a new method of modeling evolutional systems interacting with medium. A cutting process or tribo-space formed in the contact area of two conjugate mechanical subsystems is 
considered as medium. The features of the medium depend not only on state coordinates of systems but also on trajectories. It is obtained that the parameters of the medium are performed as integral operators of equations like Volterra equations of $2^{\text {nd }}$ type. The problems of control of evolutional systems are being analyzed.

\section{MATHEMATICAL MODEL OF EVOLUTIONAL SYSTEM INTERACTING WITH THE CUTTING PROCESS}

While creating dynamic models of controlled systems the hierarchy principle is used. It's based on separating motions onto "slow" ones of executive elements and "rapid" ones, which are considered in variations towards the trajectories of "slow" motions [7, 8]. Let's consider a set of trajectories of "slow" motions of the executive elements, which are being formed in the independent reading system $X=\left\{X_{1}, X_{2}, X_{3}, X_{4}\right\}^{T} \in \mathbf{X}$. For example, in conformity with turning processing components of vector $X$ have following meanings: $X_{1}$ - coordinate of the cross-moving support; $X_{2}$ - coordinate of support moving towards the cutting speed (it's obvious that in the traditional version of the machine $X_{2} \equiv 0$ ); $X_{3}$ coordinate of the support cross-moving; $X_{4}$ - angle coordinate of the spindle position. If while processing the frequency of spindle rotation is kept constant, $d X_{4} / d t=$ const. As follows, the trajectories of executive machine motions are a set in the $\mathbf{X}$ space. One of the most important of them is cutting forces $F=\left\{F_{1}(t), F_{2}(t), F_{3}(t)\right\}^{T} \in \mathbf{F}$, settled with their own projections. The cutting forces work while some of their power leads to changing of properties of the cutting process, causing the evolutional transformations of the dynamic cutting system.

Cutting forces depend on elastic tool deformations towards the part. That is why let's add the vectors of elastic tool deformations $Y(t)=\left\{Y_{1}(t), Y_{2}(t), Y_{3}(t)\right\}^{T} \in \mathbf{Y}$ towards the part $Z(t)=\left\{Z_{1}(t), Z_{2}(t), Z_{3}(t)\right\}^{T} \in \mathbf{Z}$. As follows, in general case the cutting forces are functions of all coordinates of $\mathbf{X}, \mathbf{Y}$ and $\mathbf{Z}$ spaces. As already noticed, within this article the trajectories of executive machine elements and geometry of the part in the $\mathbf{X}$ space are considered to be already settled. Following by [9], let's define the equation of dynamics of the system, which should also contain the evolutionary changes of parameters $p=\left\{p_{1}, p_{2}, \cdots, p_{s}\right\}$ of dynamical characteristic of the cutting process (in general case medium).

$$
\begin{aligned}
& m \frac{d^{2} Y(t)}{d t^{2}}+h \frac{d Y(t)}{d t}+c Y(t)=F\left(X, \frac{d X}{d t}, Y, \frac{d Y}{d t}, Z, \frac{d Z}{d t}, p\right), \\
& M \frac{d^{2} Z(t)}{d t^{2}}+H \frac{d Z(t)}{d t}+C Z(t)=F\left(X, \frac{d X}{d t}, Y, \frac{d Y}{d t}, Z, \frac{d Z}{d t}, p\right), \\
& p^{(i)}(A)=p_{i, 0}+p_{i} \int_{0}^{A} w_{P_{i}}(A-\tau) N(\tau) d \tau, \quad i=1,2 \ldots s, \\
& A(t)=\int_{0}^{t} N(t) d t, \quad N(t)=V_{p}\left|F_{2}(t)\right|,
\end{aligned}
$$


where $m, M$ are diagonal matrixes sized $3 \times 3 ; c=\left[c_{s, k}\right], C=\left[C_{s, k}\right]$ - positively defined symmetrical matrices of rigidity of subsystem of the instrument and part sized $3 \times 3$, nonchangeable at coordinates of support motions and non-changeable while changing the position of the point of system equilibrium; $h=\left[h_{s, k}\right], H=\left[H_{s, k}\right]$ - positively defined symmetrical matrices of dissipation of the subsystem instrument and part sized $3 \times 3$, also non-changeable at coordinates of support motions and non-changeable while changing the position of the point of system equilibrium; $w_{P_{i}}(A-\tau)=\exp \left[-\frac{1}{T_{P_{i}}}(A-\tau)\right]$ - kernels of the integral operators, while $T_{P_{i}}$ - constant works, which are estimated in $\mathrm{kgm}$. These parameters characterize the evolutional trajectories heredity while working. So, the evolutional heredity is displayed only within the integral operator kernel attenuation while working motion is negative (taking into consideration only the pre-history of power on work done); $V_{P}$ - cutting speed, which in our case is considered to be constant.

At the beginning let's observe the system, which possesses features [9]:

- Cutting forces satisfy the hypothesis about their constant orientation in space. This orientation is set by angular coefficients $\chi=\left(\chi_{1}, \chi_{2}, \chi_{3}\right)$.

- In variations concerning a stationary trajectory, which is assigned by $X$, the dynamic characteristic can be linearized while the reaction from the side of processing can be replaced by matrixes of the dynamic rigidity and dissipation. Then instead of (1) we have:

$$
\begin{aligned}
& m \frac{d^{2} Y(t)}{d t^{2}}+h \frac{d Y(t)}{d t}+c Y(t)=F(t)\left\{\chi_{1}, \chi_{2}, \chi_{3}\right\}, \\
& M \frac{d^{2} Z(t)}{d t^{2}}+H \frac{d Z(t)}{d t}+C Z(t)=F(t)\left\{\chi_{1}, \chi_{2}, \chi_{3}\right\}, \\
& F(t)=F_{0}+F^{(E)}(t)+\alpha(A)\left(-Y_{1}(t)-Z_{1}(t)\right)+\beta(A)\left(-\frac{d Y_{1}}{d t}(t)-\frac{d Z_{1}}{d t}(t)\right), \\
& F^{(E)}(t)=f \int_{0}^{A} w_{\alpha}(A-\tau) N(\tau) d \tau, \quad \alpha(A)=\alpha_{0}+\gamma \int_{0}^{A} w_{\alpha}(A-\tau) N(\tau) d \tau, \\
& \beta(A)=\beta_{0}+\eta \int_{0}^{A} w_{\beta}(A-\tau) N(\tau) d \tau, \quad A(t)=\int_{0}^{t} N(t) d t, \quad N(t)=V_{p}\left|\chi_{2} F(t)\right|,
\end{aligned}
$$

where $f, \gamma, \eta$ - balanced coefficients, which have, respectively, the dimension $\left[\mathrm{s} / \mathrm{kgm}^{2}\right]$, $[\mathrm{s}$ $\left./ \mathrm{kgm}^{3}\right]$ and $\left[\mathrm{s}^{2} / \mathrm{kgm}^{3}\right]$. These coefficients characterize the intensity of evolutional changes of constant components of cutting force, rigidity and dissipation of processing. In this case evolutional components of constant component of cutting force, rigidity coefficient and dissipation are, respectively, measured in $[\mathrm{kg}],[\mathrm{kg} / \mathrm{m}]$ and $[\mathrm{kgs} / \mathrm{m}]$.

In the system (2) initial conditions are determined by the values of elastic tool deformations $Y^{*}=\left\{Y_{1}^{*}, Y_{2}^{*}, Y_{3}^{*}\right\}^{T}$ concerning detail $Z^{*}=\left\{Z_{1}^{*}, Z_{2}^{*}, Z_{3}^{*}\right\}^{T}$ at initial stage of process, when evolutional changes of parameters of dynamic rigidity and dissipation are absent, i. e. $\alpha(A)=\beta(A)=0$. Thus,

$$
Y(0)=\left\{Y_{1}^{*}, Y_{2}^{*}, Y_{3}^{*}\right\}^{T}, \frac{d Y(0)}{d t}=\{0,0,0\}^{T}, Z(0)=\left\{Z_{1}^{*}, Z_{2}^{*}, Z_{3}^{*}\right\}^{T}, \frac{d Z(0)}{d t}=\{0,0,0\}^{T} .
$$


In the given model the speed of cutting is considered to be constant. Thus, if system has the point of equilibrium, which is asymptotical steady, the work is made only by the force component, directed by cutting speed. The coordinates $Y(0)=Y^{*}$ and $Z(0)=Z^{*}$, which determine the initial conditions of system, are the results of systems

$$
c Y(0)=F(0)\left\{\chi_{1}, \chi_{2}, \chi_{3}\right\}^{T}, \quad C Z(0)=F(0)\left\{\chi_{1}, \chi_{2}, \chi_{3}\right\}^{T}
$$

Functions $Y$ and $Z$ characterize the displacement of a point of equilibrium, caused by features of powered interaction at initial stage of a cutting process and evolutional transformations of system (2) due to the work, which is made at some power of irreversible transformations, depending on trajectory. Beginning of evolutional transformations is at point $t=0$, where the finished work equals zero. That is why during analysis it is reasonable to examine composition $Y(A)=Y^{*}+Y^{(E)}(A)$ and $Z(A)=Z^{*}+Z^{(E)}(A)$. This composition corresponded in time with the other one $Y(t)=Y^{*}+Y^{(E)}(t)$ and $Z(t)=Z^{*}+Z^{(E)}(t)$. As work, time, current forces and motions in (2) are interconnected, we may also observe displacement functions $Y\left(X_{3}\right)=Y^{*}+Y^{(E)}\left(X_{3}\right)$ and $Z\left(X_{3}\right)=Z^{*}+Z^{(E)}\left(X_{3}\right)$ of the point of equilibrium by moving of instrument relatively to work piece. Here $X_{3}$ - is the way, which is made by the instrument towards the work piece during manufacturing products.

Function $A(t)$ increases at all interval of integration, as subintegral expression is non-negative at any moment of time. As in the resulted simplified model rigidity and dissipation matrices have coefficients, which "slowly" change in course of evolutional transformations, thus not only the point of equilibrium is evolutional. Dynamic properties of system for "fast" movements also change. That's why roots trajectories of characteristic polynomial in complex plane should correspond to evolutional trajectories $Y^{(E)}(t)$ and $Z^{(E)}(t)$. However, at high intensity of evolutional transformations the situation when evolutional transformations themselves may influence the dynamic of system is possible. The intensity of evolutional transformations in (2) is determined by coefficients $\gamma$ and $\eta$.

Table 1. Tool subsystem parameters

\begin{tabular}{|cc|cc|c|c|}
\hline$m \cdot 10^{-3}, \mathrm{kgs}^{2} / \mathrm{mm}$ & \multicolumn{2}{|c|}{$h, \mathrm{kgs} / \mathrm{mm}$} & $c \cdot 10^{3}, \mathrm{~kg} / \mathrm{mm}$ \\
\hline$\left[\begin{array}{ccc}0,25 & 0 & 0 \\
0 & 0,25 & 0 \\
0 & 0 & 0,25\end{array}\right]$ & {$\left[\begin{array}{ccc}0,6 & 0,1 & 0,08 \\
0,1 & 0,5 & 0,2 \\
0,08 & 0,2 & 0,7\end{array}\right]$} & {$\left[\begin{array}{ccc}1,0 & 0,3 & 0,2 \\
0,3 & 1,2 & 0,4 \\
0,2 & 0,4 & 1,6\end{array}\right]$} \\
\hline
\end{tabular}

Table 2. Work-piece subsystem parameters

\begin{tabular}{|cc|c|c|c|}
\hline$m \cdot 10^{-3}, \mathrm{kgs}^{2} / \mathrm{mm}$ & \multicolumn{2}{|c|}{$h, \mathrm{kgs} / \mathrm{mm}$} & \multicolumn{2}{|c|}{$c \cdot 10^{3}, \mathrm{~kg} / \mathrm{mm}$} \\
\hline$\left[\begin{array}{ccc}5,0 & 0 & 0 \\
0 & 5,0 & 0 \\
0 & 0 & 5,0\end{array}\right]$ & {$\left[\begin{array}{cccc}5,0 & 1,0 & 0,8 \\
1,0 & 4,0 & 0,6 \\
0,8 & 0,6 & 2,0\end{array}\right]$} & {$\left[\begin{array}{ccc}0,2 & 0,1 & 0,08 \\
0,1 & 0,2 & 0,01 \\
0,08 & 0,01 & 1,0\end{array}\right]$} \\
\hline
\end{tabular}

Let's give an example of the displacement of balanced point of system for shaft turning, parameters of work-piece dynamic model are constant. It's important to note, that the displacement of balanced point corresponds to a change of work-piece's diameter. Besides we'll consider dynamical characteristics of tool subsystem to be constant, as all 
processing conditions, except values of dynamic rigidity and dissipation of cutting process. The main parameters are listed in Tables 1,2 , initial values of rigidity and dissipation equal, respectively, $\alpha_{0}=100 \mathrm{~kg} / \mathrm{mm}$ and $\beta_{0}=10 \mathrm{kgs} / \mathrm{mm}$. Cutting force at initial stage $F_{0}=100 \mathrm{~kg}$. Evolutional hereditary constants $T_{\alpha}$ and $T_{\beta}$ equal to, respectively, $T_{\alpha}=50 \mathrm{kgm}$ and $T_{\beta}=20 \mathrm{kgm}$. Force orientation coefficients: $\chi_{1}=0,50, \chi_{2}=0,71, \chi_{3}=0,50$.

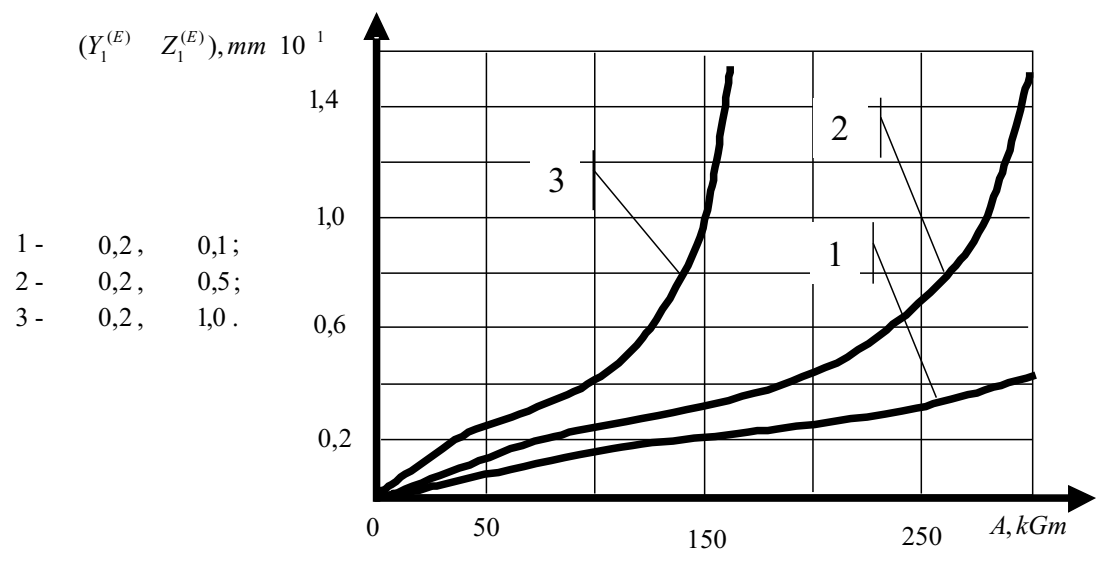

Fig. 1. Displacement of system point of equilibrium in direction, normal to workpiece rotation axis, with it's evolutional transformations

We see that depending on parameters of intensity of evolutional transformations $\gamma$ and $\eta$, displacement curves of a balanced point in the direction, normal to work-piece rotation axis, change (see Fig. 1) and remind tool wear curves in some of their values. This specifies again at relation of tool wear value with changes of dynamical characteristic of cutting process. Let's examine root trajectories of the characteristic polynomial (Fig. 2a), which correspond to deviation evolutional trajectories of a balanced point, shown in Fig. 1.

Trajectories are calculated for the system characteristic polynomial (2) assuming, that current parameters of system are frozen. This system has six pairs of complexconjugated roots $p_{i}^{(1)}=-a_{i}^{(1)}+j b_{i}^{(1)}$ and $p_{i}^{(2)}=-a_{i}^{(1)}-j b_{i}^{(1)}(i=1,2, \cdots, 6)$, which are located at left complex half-plane, because the frozen system is steady at all observed functioning segment. Evolutional displacement of balanced point corresponds with evolutional trajectory of every root or its' material and imaginary components. In Fig. 2b an example of separate evolutional stages division by classes with indication of balanced point is shown. Estimation of $Y^{(E)}(t)$ and $Z^{(E)}(t)$ may be absolutely exact, because an observed system is determined and evolutional roots curves have no ambiguity sections. But a question about the evolutional displacement estimation may arise based on autoregressive spectral analysis with the observation at vibrating succession. In this case an informational space may be formed, which consisted of material and imaginary roots components. Classification methods, which were earlier examined also, may be used, for example at the base of Baies classification rule [10]. In this example we accept the hypothesis about invariability of cutting forces orientation in space, which is correct only in 
low-frequency field and at wear small variations of cutting tool. While examining the system (1) in variations, we should notice that during evolution redistribution between force components, operated in different directions, is observed. Moreover in summarized rigidity and dissipation matrixes, not only skew-symmetric components are formed, but also matrixes themselves may become negatively determined. In this case the point of equilibrium may become unstable. After this, in condition space some varieties are formed. In this case system properties, including indexes of products geometrical quality, become dependent on initial conditions and small indignations, which influence the system. It's important to note, that because of skew-symmetric rigidity and dissipation matrixes influence at performed work and power during periodic tool movements relatively to workpiece, variable components of cutting forces, which have difficult orientation in space, start working. The work is made by force components, caused by skew-symmetric terms of rigidity matrix and symmetric terms of dissipation matrix. In this case the work is examined along the trajectory of periodical tool movements towards the workpiece.

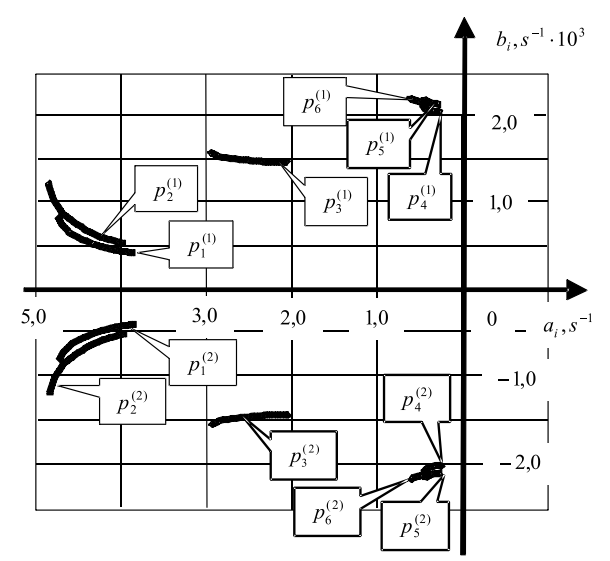

(a)

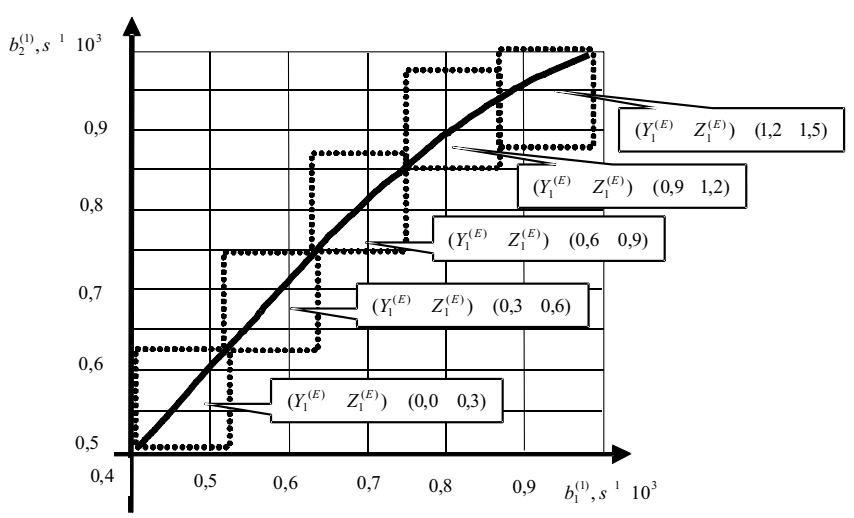

(b)

Fig. 2. Evolutional roots trajectories of characteristic polynomial (a) and example of division by classes with the value of evolutional displacement of point of equilibrium (b)

Let's illustrate this evolutional mode at a simple example. We'll consider a part to be not deformed, and turning process at constant external conditions (speed, delivery, allowance). For simplification we won't examine the influence of work and power at general cutting forces, i.e. $F^{(E)} \equiv 0$. In this way only dynamic rigidity and dissipation matrices of process are evolutionary changed. Tool deformations in direction of $X_{1}$ influence the quasistatic positional forces in variations relatively to the balanced point $X^{*}$. These components change summarized matrix of system rigidity. Concerning the dissipation matrix of cutting process, in result of evolution, as we marked earlier, all its' components are being changed. Cutting speed and two force components $F_{0}=\left\{F_{0,1}, F_{0,2}, F_{0,3}\right\}^{T}$ are considered to be constant. In that way, instead of (3) we have the following system: 


$$
\begin{aligned}
& m \frac{d^{2} X(t)}{d t^{2}}+h \frac{d X(t)}{d t}+c X(t)=F\left(X, \frac{d X(t)}{d t}\right), \\
& F\left(X, \frac{d X(t)}{d t}\right)=\left\{F_{1}\left(X, \frac{d X(t)}{d t}\right), F_{2}\left(X, \frac{d X(t)}{d t}\right), F_{3}\left(X, \frac{d X(t)}{d t}\right)\right\}^{T}, \\
& F_{i}\left(X, \frac{d X(t)}{d t}\right)=F_{0, i}+\alpha_{i}\left(-X_{1}(t)\right)+\beta_{i}\left(-\frac{d X_{1}}{d t}\right)+\phi_{i}\left(-\frac{d X_{2}}{d t}\right)+\mu_{i}\left(-\frac{d X_{3}}{d t}\right), \\
& \alpha_{i}(A)=\alpha_{0, i}+\alpha_{i} \int_{0}^{A} w_{\alpha, i}(A-\tau) N(\tau) d \tau, \quad \beta_{i}(A)=\beta_{0, i}+\beta_{i} \int_{0}^{A} w_{\beta, i}(A-\tau) N(\tau) d \tau, \\
& \phi_{i}(A)=\phi_{0, i}+\phi_{i} \int_{0}^{A} w_{\phi, i}(A-\tau) N(\tau) d \tau, \quad \mu_{i}(A)=\mu_{0, i}+\mu_{i} \int_{0}^{t} w_{\phi, i}(A-\tau) N(\tau) d \tau, \\
& A(t)=\int_{0}^{t} N(t) d t, \quad N_{i}(t)=V_{p}\left|F_{2}(t)\right| .
\end{aligned}
$$

Operator kernels in (5) have the same structure as in (2). But performed work corresponds to (2) only in the case, when system has asymptotically steady point of equilibrium. In general case comparatively from (2) work and power are determined for forces, which have different projections at axes $X_{1}, X_{2}$ and $X_{3}$. Expressions for calculation of work in general case are carefully analyzed in our monograph [9]. Matrix $m$ in system $(5)$ is diagonal. Concerning elasticity $c^{(R)}$ and dissipation $h^{(R)}$ matrices, rigidity matrix, taking into account reaction from the side of cutting process, equals:

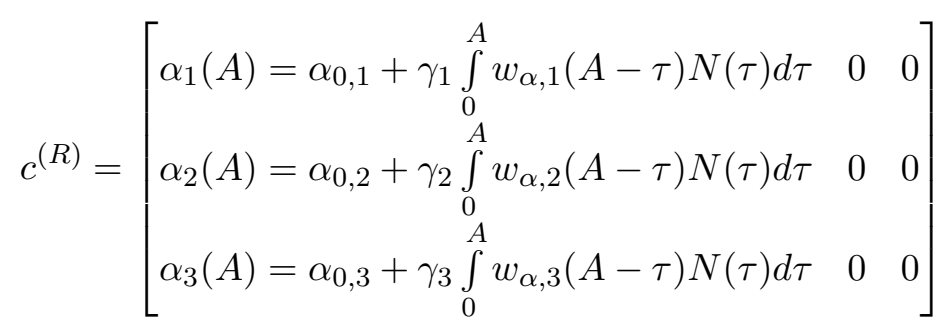

And dissipation matrix of cutting process equals:

$$
h^{(R)}=\left[\begin{array}{lll}
\beta_{1}(A) & \phi_{1}(A) & \mu_{1}(A) \\
\beta_{2}(A) & \beta_{2}(A) & \mu_{2}(A) \\
\beta_{3}(A) & \phi_{3}(A) & \mu_{3}(A)
\end{array}\right]
$$

with

$$
\begin{aligned}
& \beta_{1}(A)=\beta_{0,1}+\beta_{1} \int_{0}^{A} w_{\beta, 1}(A-\tau) N(\tau) d \tau, \phi_{1}(A)=\phi_{0,1}+\phi_{1} \int_{0}^{A} w_{\phi, 1}(A-\tau) N(\tau) d \tau, \\
& \mu_{1}(A)=\mu_{0,1}+\mu_{1} \int_{0}^{A} w_{\mu, 1}(A-\tau) N(\tau) d \tau, \beta_{2}(A)=\beta_{0,2}+\beta_{2} \int_{0}^{A} w_{\beta, 2}(A-\tau) N(\tau) d \tau,
\end{aligned}
$$




$$
\begin{aligned}
& \mu_{2}(A)=\mu_{0,2}+\mu_{2} \int_{0}^{A} w_{\mu, 2}(A-\tau) N(\tau) d \tau, \beta_{3}(A)=\beta_{0,3}+\beta_{3} \int_{0}^{A} w_{\beta, 3}(A-\tau) N(\tau) d \tau, \\
& \phi_{3}(A)=\phi_{0,3}+\phi_{3} \int_{0}^{A} w_{\phi, 3}(A-\tau) N(\tau) d \tau, \mu_{3}(A)=\mu_{0,3}+\mu_{3} \int_{0}^{A} w_{\mu, 3}(A-\tau) N(\tau) d \tau .
\end{aligned}
$$

In such a way, during evolution we observe elements redistribution of rigidity matrix and dissipation of cutting system. Besides in rigidity matrices, as a rule, those components, which form force reactions from the cutting process side in directions $X_{1}$ and $X_{3}$, increase. Coefficients of dissipation matrix vary as a result of next factors action:

- Essentially they depend on the value of delayed argument with forming of cutting forces modifications. Delayed argument at constant cutting speed grows during the volume increasing of plastic deformation in the cutting area, involved into the system reorganization. That's why dissipation matrixes at small variations of condition coordinates relatively to the balanced point may be negative and as a rule they increase on module during the evolutional modifications;

- Because of kinetic characteristic of a cutting process at fallen section of forces' dependence on speed, an effect of negative friction in the field of tool front side contact with shaving and in a field, which adjoin to rear surface of an instrument is observed. As a rule this effect increases according to wear-in and wearing development, i.e. during the evolution of system.

Let's examine the sample of evolutional modification of a cutting system in supposition, that dissipation matrices are constant, i.e. $\beta_{i}=\phi_{i}=\mu_{i}=0$. We examine only case, when the work, which is made while system vibrations around the point of equilibrium is negligible small in comparison with the work which is made by the main component of a cutting force as far as moving of tool towards the workpiece. This is fair in those cases when balanced point is asymptotically steady or varieties, which are formed in the vicinity of this point, have amplitudes, which are much smaller than the value of allowance on processing. Then instead of (5) we should examine the next simplified evolutional cutting system:

$$
\begin{aligned}
& m \frac{d^{2} X(t)}{d t^{2}}+h_{\Sigma} \frac{d X(t)}{d t}+c X(t)=F\left(X, \frac{d X(t)}{d t}\right), \\
& F\left(X, \frac{d X(t)}{d t}\right)=\left\{F_{1}\left(X, \frac{d X(t)}{d t}\right), F_{2}\left(X, \frac{d X(t)}{d t}\right), F_{3}\left(X, \frac{d X(t)}{d t}\right)\right\}^{T}, \\
& F_{i}\left(X, \frac{d X(t)}{d t}\right)=F_{0, i}+\alpha_{i}(A)\left(-X_{1}(t)\right), \quad i=1,2,3, \\
& \alpha_{i}(A)=\alpha_{0, i}+\alpha_{i} \int_{0}^{A} w_{\alpha, i}(A-\tau) N(\tau) d \tau, \quad A(t)=\int_{0}^{t} N(t) d t, \quad N_{i}(t)=V_{p}\left|F_{2}(t)\right|,
\end{aligned}
$$

where

$$
h_{\Sigma}=\left[\begin{array}{lll}
\left(h_{1,1}+\beta_{0,1}\right) & \left(h_{2,1}+\phi_{0,1}\right) & \left(h_{3,1}+\mu_{0,1}\right) \\
\left(h_{1,2}+\beta_{0,2}\right) & \left(h_{2,2}+\phi_{0,2}\right) & \left(h_{3,2}+\mu_{0,2}\right) \\
\left(h_{1,3}+\beta_{0,3}\right) & \left(h_{2,3}+\phi_{0,3}\right) & \left(h_{3,3}+\mu_{0,3}\right)
\end{array}\right]
$$


- summarized dissipation matrix with the reaction from the side of cutting process.

Let's examine an example for this case. Main parameters of system are listed in Table 3. Summarized dissipation matrix is conditionally accepted to be symmetrical and positively definite. Initial forces values, determined by technological regimes $\left(t_{R}=2,5 \mathrm{~mm}\right.$, $S_{R}=0,2 \mathrm{~mm} / \mathrm{r}, V_{R}=120,0 \mathrm{~m} / \mathrm{min}$ ) while steel turning $20 \mathrm{X}$ by cutting plates of T15K 6 are equal to $F_{0,1}=40,0 \mathrm{~kg}, F_{0,2}=100,0 \mathrm{~kg}, F_{0,3}=60,0 \mathrm{~kg}$. Initial conditions of rigidity of cutting process coefficients are equal to, respectively, $\alpha_{0,1}=100 \mathrm{~kg} / \mathrm{mm}, \alpha_{0,2}=80,0 \mathrm{~kg} / \mathrm{mm}$, $\alpha_{0,3}=20,0 \mathrm{~kg} / \mathrm{mm}$.

Table 3. Parameters of subsystem of instrument with regard of cutting process feedback

\begin{tabular}{|cc|cc|c|c|}
\hline$m \cdot 10^{-3}, \mathrm{kgs}^{2} / \mathrm{mm}$ & \multicolumn{2}{|c|}{$h_{\Sigma}, \mathrm{kgs} / \mathrm{mm}$} & \multicolumn{2}{|c|}{$c \cdot 10^{3}, \mathrm{~kg} / \mathrm{mm}$} \\
\hline$\left[\begin{array}{ccc}0,25 & 0 & 0 \\
0 & 0,25 & 0 \\
0 & 0 & 0,25\end{array}\right]$ & {$\left[\begin{array}{ccc}1,2 & 0,2 & 0,1 \\
0,2 & 1,5 & 0,2 \\
0,1 & 0,2 & 1,4\end{array}\right]$} & {$\left[\begin{array}{ccc}1,0 & 0,5 & 0,2 \\
0,5 & 1,2 & 0,4 \\
0,2 & 0,4 & 1,6\end{array}\right]$} \\
\hline
\end{tabular}

As earlier let's study the evolutional diagrams (Fig. 3) of displacement of the point of equilibrium of the system $X(t)=X^{*}+X^{(E)}(t)$ and respective diagrams of displacement of the roots of characteristic polynomial while the initial values of the system (8) are equal $X(0)=\left\{\begin{array}{ll}0,06 m m & 0,12 m m 0,09 m m\end{array}\right\}^{T}, d X(0) / d t=\left\{\begin{array}{lll}0 & 0 & 0\end{array}\right\}^{T}$.

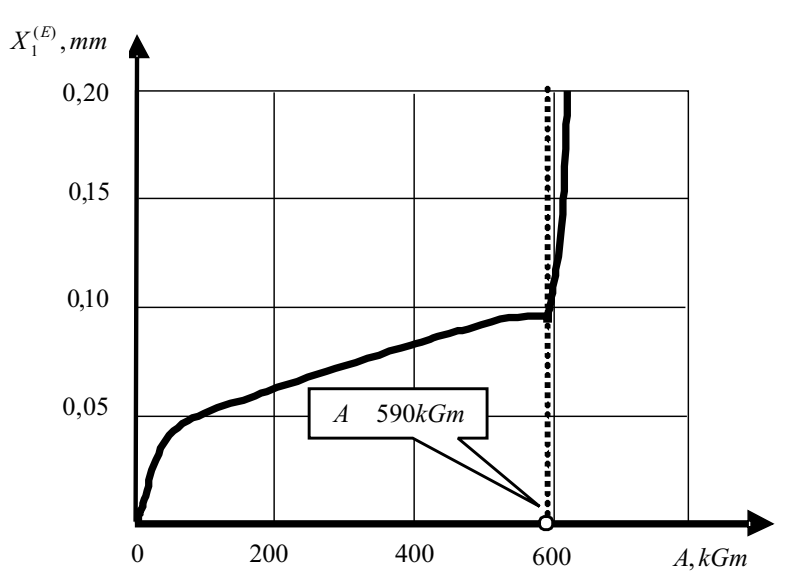

(a)

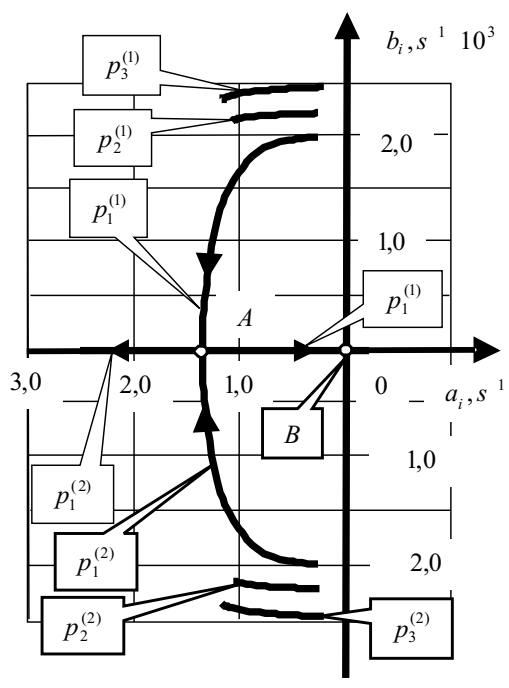

(b)

Fig. 3. Evolutional trajectories of displacement of the point of equilibrium of the system towards $X_{1}$ (a) and roots of characteristic polynomial (b) for the system (8)

We can see that a couple of roots of characteristic polynomial after some evolution become equal to each other (point "A" in Fig. 3b). After that the roots take different directions on material axis and one of the roots crosses the imaginary axis. At the moment "frozen" system looses the steadiness of the point of equilibrium and $X_{1}$ coordinate 
according to the law of unstable exponent leaves to eternity. So called instrument "undermining" takes place. In that case the whole system endures the double bifurcation transformations. Three laid on each other steady focuses at the initial stage of evolution are being transformed in the point "A" in Fig. 3 into two steady focuses, corresponded with vibrations of the relative knot, which asymptotically strives towards the point of equilibrium. Then in the point one of the trajectories becomes unstable, but all other trajectories strive towards it. The example proves that evolutional transformation while the cutting process are not only the instrument wear and (or) changes of the current values of diameter of the processed detail. These are only two external displays of the evolutional system transformations. In our opinion changing of the topology of the phase space, which appears while bifurcation transformations and related changes of the dynamic cutting process characteristic is worth more attention.

One more sample of the evolutional transformations of the linearized system. Let's consider the evolutional transformations to have only the dissipation matrixes as $\alpha_{i} \equiv 0$, but $\beta_{i} \neq 0, \phi_{i} \neq 0, \mu_{i} \neq 0$. As follows, let the described above system has the evolutional transformations coefficients' matrix correspond to Table 4.

Table 4. Values of coefficients of evolutional transformations of the cutting process dissipation matrix

\begin{tabular}{|c|c|c|c|}
\hline$i$ & $\beta_{i}, \mathrm{~s} / \mathrm{m}^{2}$ & $\phi_{i}, \mathrm{~s} / \mathrm{m}^{2}$ & $\mu_{i}, \mathrm{~s} / \mathrm{m}^{2}$ \\
\hline$i=1$ & $-0,02$ & 0,001 & 0,01 \\
\hline$i=2$ & 0,01 & 0,002 & 0,005 \\
\hline$i=3$ & $-0,02$ & 0,002 & 0,005 \\
\hline
\end{tabular}

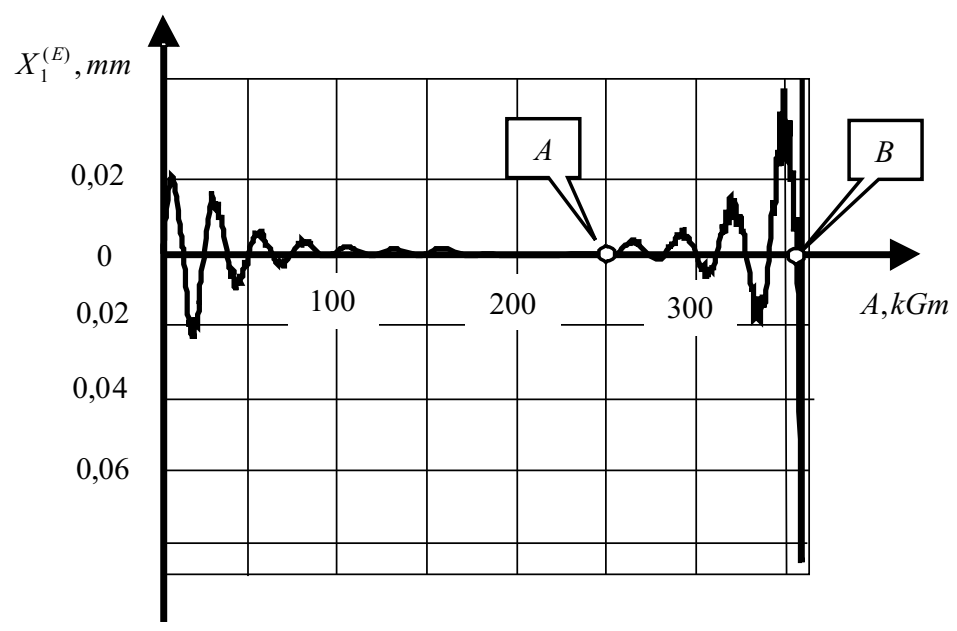

Fig. 4. Evolutional diagram of displacement of the point of equilibrium by changing the dissipation matrixes 
Let's study the sample of changing the evolutional trajectory of the point of equilibrium, calculated for the case (Fig. 4). As it was shown earlier, the elements of the matrix of dynamic dissipation of the cutting process around the point of equilibrium can have negative values. The reasons of the effect are of two kinds. Firstly, the negative values of some coefficients can be caused by lagging argument in the functions of force transformations while varying the coordinates, which affect the forces. Secondly, the negative values while the cutting speed is settled can be caused by kinetic characteristic of the friction speed in the contact area of shaving and the front surface of the instrument and in the contact area of the rear side and the detail. Both these factors increase the affecting while the wear grows during the evolutional transformations of the cutting system.

One noticeable point is that super low-frequency periodical displacements of the point of equilibrium, which go out slowly and the current values of size do set on some permanent level. So, the evolutional transformations themselves, which are modeled as the integral operators in this case, cause the behavior of the system if the system is considered to be frozen, i.e. the dynamics of the evolutional transformations affects the permanent trajectory, which can be stable or non-stable. In the highlighted sample the permanent mode is set before the work of $(250-260) \mathrm{kgm}$ (point "A" in Fig. 4) is done. Further these vibrations arise and starting from the point " $\mathrm{A}$ " the frozen dynamic cutting system looses the steadiness of the point of equilibrium. At the same time the couple of complexconjugated roots of the characteristic polynomial of the frozen system leave for the right complex semi-surface (not shown in Fig. 4). It's significant, that even small variations $\beta_{i}$, $\phi_{i}, \mu_{i}$ can lead to serious dynamic reconstruction of the system. And as the last time the topology of the phase space of the cutting system changes.

While the couple of roots of characteristic polynomial leave for the right complex semi-surface, linearized models become unacceptable. While the amplitude of periodical motions increases within the system the extra-relations, which limit the development of periodical movements are being formed. That is why it's necessary to note the non-linear dynamic models. Here let's study the case when non-linear characteristic of the cutting process is performed as suggested by Rayleigh:

$$
F^{(D)}(t)=\beta_{1} v-\beta_{2} v^{3}
$$

where $v=\frac{d Y_{1}}{d t}+\frac{d Z_{1}}{d t}$. So, let's take only the case, when the variations of displacements of the instrument towards the part only at $X_{1}$ coordinate cause the changes of the major force component, which is separated into its' own projections by matrix of angle coefficients in space.

That's why let's have a look at system (2) again. Total displacements in $X_{1}$ direction are determined as $Y_{1}+Z_{1}$. But despite of (2) let's estimate the dynamic characteristic of the cutting process on vibration speeds of the instrument towards the part in non-linear view. Also will consider the initial values of displacements $Y_{1}$ and $Z_{1}$ equal to zero, so that the system state coordinates we'll consider in the variations towards the static set of the cutting instrument in the independent counting system of the metal cutting machine. So the evolutional equation of dynamics can be performed as 


$$
\begin{aligned}
& m \frac{d^{2} Y(t)}{d t^{2}}+h \frac{d Y(t)}{d t}+c Y(t)=F(t)\left\{\chi_{1}, \chi_{2}, \chi_{3}\right\} \\
& M \frac{d^{2} Z(t)}{d t^{2}}+H \frac{d Z(t)}{d t}+C Z(t)=F(t)\left\{\chi_{1}, \chi_{2}, \chi_{3}\right\}, \\
& F(t)=F_{0}+\alpha(A)\left(-Y_{1}(t)-Z_{1}(t)\right)+\beta_{1}(A)\left(\frac{d Y_{1}}{d t}(t)+\frac{d Z_{1}}{d t}(t)\right)-\beta_{2}(A)\left(\frac{d Y_{1}}{d t}(t)+\frac{d Z_{1}}{d t}(t)\right)^{3}, \\
& \alpha(A)=\alpha_{0}+\alpha_{1} \int_{0}^{A} w_{\alpha}(A-\tau) N(\tau) d \tau, \beta_{1}(A)=\beta_{0,1}+\beta_{1} \int_{0}^{A} w_{\beta}(A-\tau) N(\tau) d \tau, \\
& \beta_{2}(A)=\beta_{0,2}+\beta_{2} \int_{0}^{A} w_{\beta}(A-\tau) N(\tau) d \tau, A(t)=\int_{0}^{t} N(t) d t, N(t)=V_{p}\left|\chi_{2} F(t)\right|,
\end{aligned}
$$

where $\beta_{0,2}$ - non-linear dissipation coefficient, measured as $\mathrm{kgs}^{3} / \mathrm{m}^{3}, \beta_{2}$ - non-linear coefficient of the evolutional transformations, measured as $\mathrm{s}^{3} / \mathrm{m}^{4}$. Initial values (10) are settled:

$$
Y(0)=\left(\begin{array}{lll}
0 & 0 & 0
\end{array}\right)^{T}, \frac{d Y(0)}{d t}=\left(\begin{array}{lll}
0 & 0 & 0
\end{array}\right)^{T}, Z(0)=\left(\begin{array}{lll}
0 & 0 & 0
\end{array}\right)^{T}, \frac{d Z(0)}{d t}=\left(\begin{array}{lll}
0 & 0 & 0
\end{array}\right)^{T} .
$$

Let's analyze the time and phase trajectories of system motion from the point (11). It's suitable to estimate them as the projection for two phase surfaces $Y, d Y / d t$ and $Z, d Z / d t$, as well as in the coordinates system, where the time value is on the abscise axis (see Fig. 5). So, the phase surface, located to the left of abscise axis, belongs to $Y, d Y / d t$, but to the right of the axis - to $Z, d Z / d t$ (see Fig. $5 \mathrm{~b}$ ). System parameters correspond with the sample mentioned earlier.

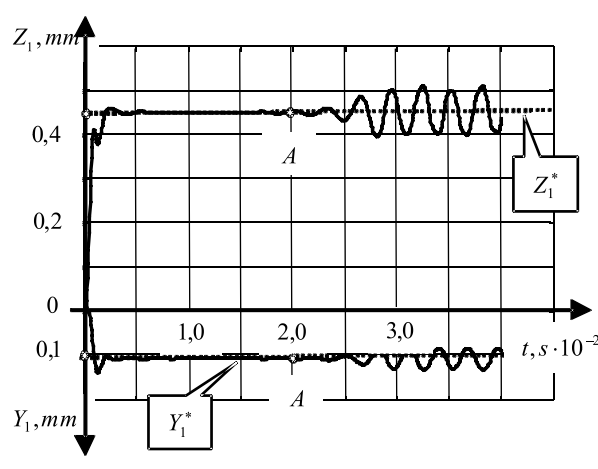

(a)

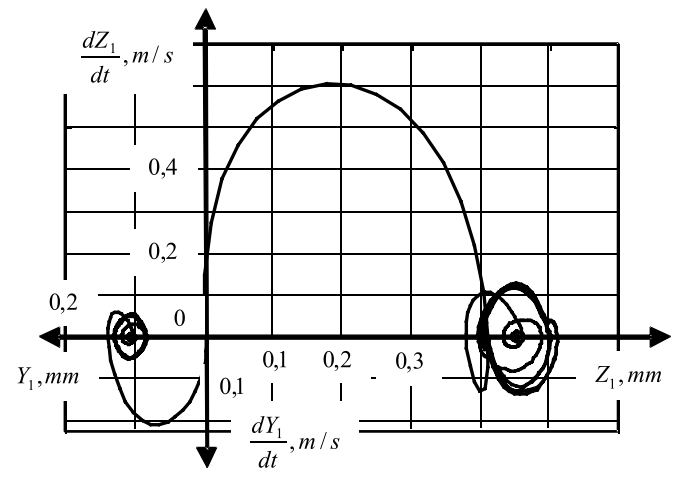

(b)

Fig. 5. Sample of evolutional diagram of instrument and detail (a) displacement for system (10) and its' phase trajectories (b) for $\alpha(A)=0$

We can see, that while $\alpha(A)=0$ after the transformation process the stationary state is being settled with the coordinates $Z_{1}=Z_{1}^{*}=0,44 \mathrm{~mm}=$ const and $Y_{1}=Y_{1}^{*}=$ 
$0,11 \mathrm{~mm}=$ const (see Fig. 5a). But with the system moving because of increasing $\beta_{1}(A)$ the roots of characteristic polynomial of the "frozen" system also move in point "A" on Fig. 5a crossing the imaginable axis. And around the $Z_{1}=Z_{1}^{*}=0,44 \mathrm{~mm}=$ const points and $Y_{1}=Y_{1}^{*}=0,11 \mathrm{~mm}=$ const dissipation matrix of the "frozen" system become negatively defined. That is why the points of equilibrium loose their steadiness and the steady limit cycle, parameters of which do change during the evolution, is being formed around the points. So, the "A" points in Fig. 5a are the points of bifurcation of AndronovKhopf. This is the bifurcation of appearing orbital asymptotically steady limit cycle from asymptotically steady point of equilibrium. If in this case to take into consideration the non-linearity in positioning relation inside the force system function, than with raising amplitude of the periodical motions the dynamic displacing of the $Y_{1}^{*}$ and $Z_{1}^{*}$ points will be noted. So, the varieties, formed in the space of "fast" motions (in our case these are the parameters of the limit cycle), affect the form generating trajectories of the "slow" motions, as follows - on current values of diameter of the being processed roller.

\section{CONCLUSION}

The above data let make following conclusions.

1. While the cutting system is on its dynamic reconstruction takes place, which is caused by evolutional transformations of parameters of dynamic characteristics of the processing. The evolutional trajectories of parameters of dynamical characteristic correspond to the trajectories of the roots of characteristic polynomial in the complex surface. Studying the trajectories of the roots within the cutting process is suitable to be worked out on the basis of autoregressive spectral analysis of the fibro-acoustic emission signal. The root trajectories characterize in their turn the information stock, which had not been used earlier, helps to diagnose and manage the cutting process online.

2. The evolution of parameters of dynamic characteristics of the cutting process causes changing of parameters of varieties, which are formed around the stationary trajectories, which are settled by trajectories of "slow" motions of executive machine elements. In some points of evolutional trajectories the changes of topology of phase space of "fast" motion subsystem are noticed, i.e. bifurcations, which change principally the dynamic mode of the cutting process. Finally, the displacing of the trajectories from the form generating motions of the instrument towards the detail is noticed. They define the values of the geometrical quality of the details. That is why studying of the trajectories of the roots of characteristic polynomial allows to estimate the current values of the geometrical quality of the details directly while processing.

\section{REFERENCES}

[1] A. M. Dalskii, Technological providing of reliability of high-precision parts of machines, Mashinostroenie, Moscow, (1975), (in Russian).

[2] K. S. Kolesnikov, Technological foundations of providing quality of machines, Mashinostroenie, Moscow, (1990), (in Russian).

[3] N. A. Bushe, Friction, wearing and fatigue in machines, Transport, Moscow, (1987), (in Russian). 
[4] V. L. Zakovorotny, Pham Dinh Tung, Nguyen Xuan Chiem, Modeling of deformation displacement of the cutting tool relatively to workpiece in turning, Herald DGTU, 10(7) (2010) 1005-1016, (in Russian).

[5] V. L. Zakovorotny, Pham Dinh Tung, Nguyen Xuan Chiem, Mathematical modeling and parametric identification of dynamic properties of the subsystems of the cutting tool and workpiece in turning, Proceedings of the Universities, The north-Caucasus region, 2 (2011) 38-47, (in Russian).

[6] A. A. Kolesnikov, Synergetic theory of control, Energoatomizdat, Moscow, (1994), (in Russian).

[7] G. Haken, Synergetics, The hierarchy of instabilities in self-organizing systems and devices, Mir, Moscow, (1985).

[8] A. N. Tihonov, Systems of the differential equations with small parameter at the higher derivatives, the Mathematical collection, 31(3) (1952) 575-586.

[9] V. L. Zakovorotny, A. D Lukyanov, Nguyen Dong Anh, Pham Dinh Tung, Synergetic system synthesis dynamic controlled machine tools, taking into account the evolution of couplings, Izd DGTU, Rostov on Don, (2008), (in Russian).

[10] V. L. Zakovorotny, E. V. Bordachev, Informational providing of a system of dynamic diagnose of wearing of cutting tool on the example of turning, Problems of engineering industry and reliability of machines, 3 (1995) 18-26, (in Russian).

Received February 9, 2011 